\title{
INSTRUMENTATION AND FIRST RESULTS OF THE REFLECTED SOLAR DEMONSTRATION SYSTEM FOR THE CLIMATE ABSOLUTE RADIANCE AND REFRACTIVITY OBSERVATORY
}

Joel McCorkel, Kurtis Thome, Jason Hair, Brendan McAndrew, Don Jennings, Douglas Rabin, Adrian Daw, Allen Lundsford

NASA's Goddard Space Flight Center

The Climate Absolute Radiance and Refractivity Observatory (CLARREO) mission key goals include enabling observation of high accuracy long-term climate change trends, use of these observations to test and improve climate forecasts, and calibration of operational and research sensors. The spaceborne instrument suites include a reflected solar spectroradiometer, emitted infrared spectroradiometer, and radio occultation receivers. The requirement for the RS instrument is that derived reflectance must be traceable to SI standards with an absolute uncertainty of $<0.3 \%$ and the error budget that achieves this requirement is described in previous work. This work describes the Solar/Lunar Absolute Reflectance Imaging Spectroradiometer (SOLARIS), a calibration demonstration system for RS instrument, and presents initial calibration and characterization methods and results. SOLARIS is an Offner spectrometer with two separate focal planes each with its own entrance aperture and grating covering spectral ranges of 320-640,600-2300 nm over a full field-of-view of 10 degrees with 0.27 milliradian sampling. Results from laboratory measurements including use of integrating spheres, transfer radiometers and spectral standards combined with field-based solar and lunar acquisitions are presented. These results will be used to assess the accuracy and repeatability of the radiometric and spectral characteristics of SOLARIS, which will be presented against the sensor-level requirements addressed in the CLARREO RS instrument error budget. 
$\sim 100$ word version for programs:

The CLARREO mission addresses the need to observe high-accuracy, long-term climate change trends and advance the accuracy of SI traceable absolute calibration. This work describes SOLARIS, a calibration demonstration system, and presents initial calibration and characterization methods and results. Results from laboratory measurements including use of integrating spheres, transfer radiometers and spectral standards combined with fieldbased solar and lunar acquisitions are presented. Results will be used to assess the accuracy and repeatability of the radiometric and spectral characteristics of SOLARIS, which will be presented against the sensor-level requirements addressed in the CLARREO RS instrument error budget. 